Derecho y Realidad

Núm. 23 • I semestre de 2014

Facultad de Derecho y Ciencias Sociales, UPTC

ISSN: 1692-3936

\title{
Cooperación, alianzas estratégicas e internacionalización de la universidad colombiana
}

\author{
Cooperation, strategic alliances and \\ internationalization of Colombian university
}

Julio Armando Rodríguez Ortega*

Resumen

Este artículo identifica una de las más importantes tendencias de la educación superior en la actualidad, asociada con la acreditación de calidad, con el prestigio de las instituciones, con la viabilidad económica de sus programas. Se materializa en la existencia de contenidos y métodos docentes, actualizados e innovadores y en la generalizada valoración y reconocimiento institucional a nivel internacional. Se precisa cómo el énfasis de dicha internacionalización se expresa fundamentalmente en la movilidad de estudiantes y docentes, mediante convenios de cooperación y el desarrollo de programas de estudio conjuntos, a través de alianzas estratégicas.

\section{Palabras clave}

Educación superior, acreditación, calidad, internacionalización, cooperación, alianzas.

* Docente de la Universidad Nacional de Colombia. 
250

Derecho y Realidad

\begin{abstract}
This article identifies one of the major trends in higher education today, associated with accreditation of quality, with the prestige of the institutions, with the economic viability of their programs. It is materialized in the existence of contents and updated and innovative teaching methods and the widespread assessment and institutional recognition at the international level. It points out how the emphasis of this internationalization is expressed primarily in the mobility of students and teachers, through cooperation agreements and the development of joint study programs through strategic alliances.
\end{abstract}

\title{
Key words
}

Higher education, accreditation, quality, internationalization, cooperation, alliances. 


\section{Introducción}

La mayor parte de las universidades han incluido en la actualidad en sus planes de desarrollo políticas institucionales de internacionalización, alianzas estratégicas para el desarrollo conjunto de programas y convenios de cooperación con múltiples entidades públicas y privadas. Con miras a lograr este objetivo se han iniciado procesos de internacionalización del currículo, incremento del bilingüismo, programas de intercambio de estudiantes y docentes, proyección internacional de los egresados, conformación y participación en redes internacionales de investigación y publicaciones conjuntas, sistemas de información y muchos otros aspectos.

Se observa en este proceso la adopción de instrumentos esenciales de valor estratégico para enfrentar exitosamente los desafíos de la globalización, mediante la cooperación internacional en la formación de profesionales con una nueva mentalidad capaces de construir una sociedad más justa y abierta basada en el respeto y en la solidaridad y en el uso compartido de los bienes del desarrollo (CNA, 2008).

La mayoría de las universidades han incluido políticas institucionales de internacionalización, situación que se refleja en dependencias encargadas de estudiantes extranjeros y en diversos mecanismos y estrategias para apoyar y facilitar convenios y alianzas estratégicas que facilitan la integración de programas y el avance en la formulación de maestrías y doctorados.

De igual forma se aprecia el esfuerzo que hacen las instituciones de educación superior para internacionalizar el currículo mediante la inclusión de pasantías y la realización de investigaciones en el extranjero. En la mayoría de los reglamentos estudiantiles de postgrado se encuentra referencia a homologación y programas con doble titulación o programas conjuntos, lo cual ofrece la posibilidad de tratar problemáticas de carácter internacional. En este sentido se ha incrementado la exigencia en el requisito de lengua extranjera con la demanda de competencias comunicativas en inglés, lecturas en lenguas extranjeras que son objeto de seminarios y cursos, y en la misma forma se aprecia con reiterada frecuencia que se ofrecen cursos en lengua extranjera en temas relevantes para los programas objeto de convenio.

El más importante indicador de la internacionalización de estudiantes y profesores se puede observar en las estadísticas de estudiantes extranjeros en la universidad colombiana tanto en la investigación como en las publicaciones. Son cada vez más numerosos los convenios de intercambios activos con universidades extranjeras y las experiencias de homologación, el número de profesores visitantes y graduados en universidades del exterior, los convenios de beca y los proyectos de financiación con fuentes de la cooperación internacional. 
Los egresados tienen en la actualidad una proyección internacional que se puede apreciar en el número de estudiantes que han sido admitidos en programas en el extranjero: maestrías, doctorados, postdoctorados, pasantías y profesores visitantes que en la actualidad son objeto de seguimiento por parte de las universidades. El más importante indicador de la internacionalización es el relacionado con la investigación, pues en la actualidad son numerosas las redes internacionales que involucran investigadores y grupos de investigación nacionales y extranjeros. Son numerosos los proyectos de investigación conjuntos con universidades o centros de investigación del exterior, tesis de doctorado o de maestría dirigidas por profesores extranjeros, acceso a laboratorios, centros de investigación y centros de información de universidades extranjeras con la participación de investigadores y estudiantes en eventos y programas internacionales.

Finalmente, la internacionalización de las universidades en esta época de globalización académica se observa de manera particular en las publicaciones y en los sistemas de información que permiten el acceso a bases de datos extranjeras por parte de estudiantes y profesores, publicaciones conjuntas con programas de otras universidades y particularmente la realización de alianzas estratégicas para el desarrollo de programas de maestría y doctorado que junto con la doble titulación dan cuenta de un proceso en el que se está consolidando el carácter internacional de las instituciones de educación superior (CNA, 2008).

\section{Cooperación internacional}

Se trabaja sobre la base de que la educación se fortalece con los principios de cooperación internacional, fundada en la solidaridad, el reconocimiento y el apoyo mutuo que permite poner en común sus potencialidades, conocimientos teóricos y prácticos, sus experiencias de progreso y sobre todo los esfuerzos por lograr la equidad y la justicia social.

La cooperación internacional ha sido un sostén importante de las instituciones de educación superior en América Latina, por cuanto ha ayudado a afianzar modelos foráneos de generación de conocimientos y a acumular valiosas experiencias que pueden ser transferidas a otras instituciones por vía de nuevos esquemas de cooperación, para asumir los retos del desarrollo. Contrario a lo anterior, se evidencian numerosos obstáculos enraizados en las mal llamadas democracias de América Latina, en las cuales la corrupción, los privilegios y la discriminación son ostensibles.

Se vislumbra la necesidad de asegurar la formación de profesionales competentes para un mundo globalizado que se inserten efectiva y positivamente en la comunidad 
internacional, obligando a las Instituciones de educación superior a retomar el fundamento inicial de la formación universitaria que implica educar ciudadanos del mundo inmersos en la búsqueda de conocimientos universales. La universidad juega un papel esencial al fortalecer la formación de hombres y mujeres capaces de interactuar globalmente dentro de un marco de convivencia ciudadana y de asumir retos profesionales en el marco de una economía abierta y globalizada

La internacionalización que durante largo tiempo fue un asunto de interés marginal, ha cambiado de sentido y en la actualidad la importancia de la internacionalización y de la cooperación como política y como actividad, ha pasado a un primer nivel, manifestándose gradualmente en un cambio en la situación. La internacionalización, y derivada de ella la cooperación, es uno de los asuntos que prevalece hoy en día en las políticas sociales y económicas, lo mismo que en la educación superior y seguramente ganará más importancia en los años por venir. Avances concretos han sido logrados en algunas partes de Latinoamérica, obviamente más en algunas que en otras.

Durante largo tiempo ha habido un consenso acerca de los beneficios de la internacionalización y de la cooperación de tal forma que algunos académicos y profesionales administrativos han creído casi ciegamente en sus beneficios en contraposición a un grupo de escépticos que siguen formulando dudas acerca de su importancia, pues casi siempre su voz se pierde en el desierto, en los nacionalismos y en las burocracias estatales. En consecuencia se ha desarrollado un debate crítico acerca de las bondades de la cooperación y de la internacionalización, aunque ciertamente estas dos variables no han logrado en muchas instituciones constituirse como un elemento central en los planes de desarrollo de la América Latina

La cooperación interregional latinoamericana, como una de las actividades de la internacionalización, contribuye eficazmente en todos los campos a lograr mejores niveles de desarrollo. El campo de la educación, por ejemplo, se ha dicho que es internacional por naturaleza, pues a través de la movilidad y el intercambio estudiantil y de docentes se trasmiten competencias claves tales como el aprendizaje de una lengua extranjera y la experiencia intercultural necesaria para funcionar de manera exitosa internacionalmente.

Se observan, sin lugar a dudas, los comienzos de un mercado competitivo de la educación superior en una escala global la cual es probable que coloque las consideraciones económicas en un lugar preponderante de la agenda al lado de los desafíos académicos tradicionales. Como resultado de lo anterior, entre un número cada vez mayor de instituciones de educación superior y la tendencia a exportar la educación como un servicio, parece ganar terreno. 
Las universidades en algunos países han concentrado su foco de interés en la actividad internacional y por lo tanto en los estudiantes extranjeros, quienes representan una importante fuente de ingresos. Algunas instituciones han perfeccionado sus estrategias de mercado internacional y desarrollan investigaciones de mercado para mejorar la dirección de sus esfuerzos de reclutamiento. En algunos países incluso las instituciones se han asociado para constituir entes que se encarguen de mercadear sus servicios educativos en el exterior (Restrepo, Sánchez \& Uribe, 2006).

Además de las consideraciones académicas y económicas, la cooperación interregional y la internacionalización de la educación superior han sido fuertemente apoyadas como un medio de la política cultural internacional. Esta situación no es solo una preocupación de las instituciones de educación superior sino también de los gobiernos. Para ellos, la cooperación en un amplio rango de campos culturales y educativos es un elemento del conjunto de la política exterior como un todo. La cooperación internacional contribuye a apoyar el interés de desarrollar no solo lazos de amistad sino que tradicionalmente las políticas culturales se expresan en acuerdos bilaterales de los gobiernos, en los cuales se identifican proyectos y actividades específicas entre las cuales se pueden mencionar la homologación y convalidación de títulos y cursos académicos.

Gracias a la cooperación y a la internacionalización de los servicios del conocimiento, se rompen las fronteras y las universidades ofrecen sus programas tanto en sus países de origen como en otros, a través de convenios de colaboración o alianzas estratégicas, y por tanto en sus funciones sustantivas de docencia, investigación y extensión, debe estar implícita la responsabilidad de formar para el mundo; así mismo es condición importante tener intrínseco el concepto de calidad en todas las acciones que se desarrollen, para ocupar una posición importante y competitiva en el mundo globalizado.

En cuanto a la internacionalización de la investigación, se mira como una proyección para solucionar problemas reales iniciando con los que contribuyan al mejoramiento del entorno, como una fuente de conocimiento que mide el nivel internacional que se tiene, y los resultados obtenidos para debatir en escenarios nacionales e internacionales, con la visión universal que deben tener las Instituciones de educación superior, bajo un esquema de internacionalización desde lo global ${ }^{1}$.

La cooperación científica y tecnología internacional, constituye actualmente un instrumento estratégico en el diseño de las políticas científicas y tecnológicas de un Estado, en el desarrollo institucional de las universidades y centros de investigación

$\overline{1}$ Para un análisis más detallado del proceso de internacionalización y de sus implicaciones, ver CNA (2007). 
y desarrollo y en la competitividad de las empresas. La cooperación es, además, un elemento intrínseco en los procesos de generación del conocimiento científico, del desarrollo tecnológico, de los procesos de difusión y transferencia tecnológica y de la innovación.

Las instituciones de educación superior definen en su política de internacionalización y cooperación a través de alianzas o convenios internacionales con el fin de desarrollar iniciativas conjuntas de colaboración que beneficien y fortalezcan la calidad académica y el desarrollo y consolidación de sus esfuerzos de internacionalización. Esta colaboración puede enmarcarse en iniciativas que promuevan la construcción de redes temáticas, el intercambio de información bibliográfica, el desarrollo de programas conjuntos y el diseño de programas de movilidad académica.

La cooperación e internacionalización es de carácter regional, si los programas de cooperación se desarrollan en una zona geográfica determinada y en ellos concurren las instituciones de educación superior (IES) para hacer operativo y dar continuidad al desarrollo educativo. Un ejemplo es el programa Sócrates, que es el resultado de la unión del programa de acción de la Unión Europea para la movilidad de los estudiantes universitarios (ERASMUS, 1987) y el programa para promover el conocimiento de lenguas extranjeras en la CE (Lingua, 1990).

En igual sentido es de carácter global, cuando las instituciones de educación superior se incorporan en programas que tienen como fin la integración y cooperación interuniversitaria a escala mundial a través de sistemas que cubren casi todos los campos del conocimiento y un número considerable de países de todas las regiones. Como ejemplo de esta relación global, está el programa UNITWIN de la UNESCO que promueve la creación o el fortalecimiento de numerosas cátedras y redes que despliegan actividades de enseñanza e investigación en múltiples campos del saber.

Los medios de cooperación interinstitucional de la educación superior se definen por intermedio de la aplicación de acciones multiculturales y bilaterales que integran grupos regionales, mediante la construcción de redes. En este sentido, los proyectos de movilidad, la internacionalización de los programas, la conectividad o sistemas de enlace y la aplicación de tecnologías de comunicación e información, constituyen las formas para que las instituciones de educación superior desarrollen acciones de cooperación basadas en "principios de interés común, respeto mutuo, credibilidad recíproca, solidaridad en la producción y aplicación del saber como apoyo al concepto de la comunidad fundada sobre la diversidad".

En la actualidad, el progreso y desarrollo de la humanidad se ha caracterizado por el incremento de las relaciones mundiales, el comercio, la política, los intercambios 
culturales, la permeabilidad entre los Estados, la nueva media y las nuevas posibilidades que el mundo ofrece, acciones que han determinado en las instituciones de educación superior la exigencia de crear nuevas condiciones de aprendizaje, enseñanza e investigación en concordancia con los adelantos del mundo globalizado.

\section{Globalización e instituciones de educación superior}

La internacionalización está basada en los procesos de apertura y en la evolución de la dinámica del mercado que hoy principalmente es una respuesta a los cambios traídos por la globalización. En el lenguaje académico, el término globalización no está dirigido a las distintas disciplinas, sino a todas las perspectivas que se abren en los discursos trasnacionales. Al principio de la década de los cincuenta se relacionaba globalización con lo económico, pero con el transcurrir de los años se vio como una oportunidad de apertura que permitiría desarrollar a la persona como ciudadano del mundo y dejar las tensiones y los conflictos creados por esta misma realidad.

El proceso más avanzado en la globalización actual, que incide directamente en la educación, es el del desarrollo de la información y las comunicaciones, que se encuentran en pleno progreso de innovación, causando profundas repercusiones en todas las esferas de la sociedad. La forma como se trasmite la información, le da el acceso a ella a cualquier individuo sin importar idioma, raza o lugar donde se encuentre.

Las instituciones de educación superior tienen el compromiso de orientar y educar fortaleciendo y creando nuevos valores, a través de la cooperación para atender los reclamos de la sociedad actual. Los cambios que se han producido en lo político, en las empresas, en la educación en sí y en la sociedad en general, refuerzan la necesidad del clima intelectual y científico de las instituciones de educación superior ya que a través de dicha información, se podrá dar el verdadero sentido y orientación a los países, ante el reto que imponen la globalización de la economía y su injerencia en la educación.

América Latina tiene como punto de partida en este proceso de internacionalización los cambios forzosos de la economía, lo social, lo político y el medio ambiente cultural, causado por las competencias globales, la integración de los mercados, el aumento de las redes de comunicación y la información que influye a través de la movilidad. Igualmente debe tener en cuenta que este es un proceso incontrolado, determinado principalmente por competencias entre fuerzas económicas a una escala global y por rápidos avances en la información y las tecnologías de la comunicación cuyo mayor impacto de la globalización, se ha dado en los cambios que permanentemente está sufriendo la educación superior para afrontar y preparar profesionales idóneos para el mundo que se le presenta a futuro. 
Así mismo, la globalización exige que las instituciones de educación superior se presenten al mundo con altos niveles de pertinencia y calidad, modernización, autonomía y gestión tanto en lo académico como en lo financiero, lo mismo que en lo relativo al desarrollo de la ciencia, la tecnología, producción y socialización, para poder competir en el entorno mundial. El hecho de vivir en una "aldea global" exige el compromiso de las instituciones de educación superior con todo su entorno.

La cooperación requiere que los actuales gobiernos con el aliento democrático de los pueblos impulsen la creación y el funcionamiento de entidades multilaterales adecuadas para encausar su esfuerzos a favor de las paz, equidad de trato entre los Estados, de procedimientos concertados para la solución de controversias, de soluciones racionales propuestas por las instituciones de educación superior con un mayor dinamismo en los proyectos económicos y sociales de integración regional.

La tarea de América Latina es, pues, promover una política que desarrolle de manera prioritaria la cooperación internacional, como un medio para promover el mejoramiento de la educación, el entendimiento entre los pueblos, la solución de sus problemas, la formación de recursos humanos y la actualización científica, y que alternativamente canalice fondos de las agencias donantes y cooperantes con las universidades en proyectos prioritarios de la región.

Los programas internacionales de cooperación científica y académica deben obedecer a prioridades nacionales e internacionales claramente formuladas; considerar la formación de recursos humanos a lo largo de todo el proceso como un elemento integral de todo el programa, para lo cual es necesario conocer las potencialidades nacionales y promover a través de las relaciones internacionales sistemas cooperativos en materia de información científica y tecnología para enfrentar la escasez de divisas y limitaciones presupuestarias contando con publicaciones periódicas y libros científicos necesarios para mantener actualizada la cooperación y evitar el regreso a épocas de atraso.

\section{Cooperación latinoamericana}

América Latina necesita ingresar resueltamente en la cultura y la civilización científica, pero el desarrollo científico y tecnológico no puede producirse independientemente del contexto social y sin el concurso integral de la cooperación internacional dentro de una política de desarrollo integral y autónomo que fomente la capacidad creativa y el espíritu científico para fortalecer la infraestructura investigativa y poder generar modelos de tecnología apropiada a nuestras realidades sociales económicas y culturales. En los procesos de desarrollo, las instituciones de educación superior han tenido que superar diferentes obstáculos, entre ellos uno de los que ha tenido mayores implicaciones y que la universidad como tal ha tenido 
que enfrentarse en las últimas décadas es la transformación masiva del sistema de educación superior. En los comienzos del siglo XXI, América Latina registra un crecimiento progresivo del desempleo, de la pobreza y de la miseria, razón por lo cual es más urgente que nunca consolidar programas de cooperación, para abordar imperativamente el crecimiento con equidad, la protección del ambiente y la construcción de la paz, pues desarrollo humano, democracia y paz son inseparables.

Sin reales programas de cooperación internacional, no es posible el desarrollo económico y social en esta particular etapa histórica caracterizada por la emergencia de un nuevo paradigma productivo basada en el poder del conocimiento y en el manejo adecuado de la información, para asumir con realismo las necesidades y carencias específicas de América Latina.

Los procesos simultáneos y a veces contradictorios de globalización, regionalización e integración por una parte, y los de marginación y fragmentación por otra parte, inciden en los programas de cooperación latinoamericana, al tiempo que el peso de la deuda externa, el incremento de las importaciones de bienes y servicios son factores generadores de desigualdad social, que los países de América Latina buscan enfrentar, mediante programas de cooperación regional o subregional como con la instrumentación de políticas sociales.

Frente a esta situación es evidente el papel que los sistemas educativos en general y la educación superior en particular, cumplen como instrumentos esenciales para enfrentar exitosamente los desafíos del mundo moderno y para formar ciudadanos capaces de formar una sociedad más justa y abierta basada en la solidaridad y respeto de los derechos humanos y el uso compartido del conocimiento y de la información. Se trata de dos elementos insustituibles para el desarrollo social el crecimiento económico, el fortalecimiento de la identidad cultural latinoamericana y la lucha mancomunada contra la pobreza.

Las instituciones educativas de América Latina deben generar en sus graduados la conciencia latinoamericana promoviendo los procesos que conduzcan a la integración regional y situando la integración cultural y educativa como bases de la integración política y económica frente a la formación de nuevos espacios económicos en el actual contexto de globalización y de regionalización.

La cooperación latinoamericana se materializará en el esfuerzo de las instituciones de educación superior para asumir con energía el proyecto de integración económica, política, social y cultural, como tarea fundamental con un tratamiento interdisciplinario de sus problemas. La cooperación no se logrará con el esfuerzo de los gobiernos, sino con las acciones silenciosas de organismos supranacionales, liderados por las instituciones de educación superior, que son las que deben esbozar 
los lineamientos, para armonizar las múltiples actividades que se están realizando en la región con miras a la cooperación, con el fin de aumentar su eficacia y eficiencia, evitar la duplicación innecesaria de esfuerzos y movilizar nuevos recursos hacia ella (Cárdenas et al., 2000).

En este sentido, la cooperación tiene como finalidad lograr la concurrencia de los diversos actores e instituciones para impulsar los cambios que respondan a los desafíos planteados en los procesos de globalización, regionalización e integración, pero sobre todo para dar una respuesta clara frente a la vulnerabilidad de las democracia y el derrumbe de los nacionalismos que aparecen como símbolo de la región.

Es necesario contar con la participación productiva y concertada de gobiernos y parlamentos, pero su liderazgo y protagonismo estará a cargo de las instituciones de educación superior, actores sociales, ONG y organismos internacionales y supranacionales interesados en mejorar la capacidad de las sociedades para hacer frente a los retos de la pobreza y la marginalización social, pero sobre todo para ayudar a generar y fortalecer proyectos educativos que respondan a los cambios profundos de la sociedad y a los desafíos identificados en cada uno de los países.

La construcción de sociedades más equitativas y humanas, como tarea prioritaria en el continente, es difícil de realizar sin el compromiso de quienes como formadores, enseñan a aprender y enseñan a pensar a los niños y jóvenes. Educadores que a través de un conocimiento profundo y reflexivo de su práctica pedagógica, facilitan la aproximación fecunda al conocimiento, las oportunidades de crecimiento autónomo y responsable y las condiciones del ejercicio de la ciudadanía.

El desarrollo de las sociedades latinoamericanas en el marco de una estrecha cooperación interregional, supone el desarrollo continuo de sus educadores, porque cambios de naturaleza tan compleja, como los cambios de mentalidad, de referentes éticos y valores, la necesidad de nuevas competencias sociales y cognoscitivas frente a los avances del conocimiento, solo son posibles por la acción de la educación y esta se materializa a través de la practica pedagógica de los educadores.

El reconocimiento de que la función social de mayor importancia en las sociedades democráticas la cumplen los educadores, los convierte en protagonistas esenciales de los procesos de cambio. Esta misión en la historia contemporánea de América Latina, implica una participación activa de los ciudadanos del continente en las deliberaciones sobre los cambios en educación y un compromiso ético y político surgido de su condición de nacionales latinoamericanos, para trabajar por las transformaciones sociales necesarias. 
En este sentido es importante destacar que en América latina solo hay una nacionalidad, materializada en numerosos Estados, que como tales deben contribuir a la construcción de una perspectiva política-educativa y cultural de la integración latinoamericana, creando espacios de reflexión y de debate sobre la teoría y la práctica integracionista, en torno a un eje temático singular que es el de las reformas y los procesos de cambio de la estructura económica social y política.

Las anteriores recomendaciones o propuestas son el resultado de una observación critica y un cuidadoso proceso de análisis colectivo, por el cual se infiere que el mayor número posible de intelectuales, académicos, empresarios, gremialistas, docentes de base, comunicadores sociales y otros representantes de la sociedad civil, contribuyen mejor que nadie a la renovación del pensamiento integracionista para generar las políticas y acciones de la integración que requieren las sociedades actuales, tomando como uno de los fundamentos de su trabajo el desarrollo de los procesos de cambio de mentalidad y sustitución de nacionalismos desintegradores.

Para hacer posible el proceso integracionista es necesario que se haga más efectiva la cooperación interregional y se sugiere que se avance en la homologación de títulos universitarios, en especial de todo lo relacionado con la formación humana y social. Se sugirió la organización de un currículo original para estudios de pregrado en el que, al menos, un $40 \%$ sea de contenidos comunes.

Si se logra esto, será posible fortalecer y ampliar los programas de pasantías, de año sabático, establecer mecanismos de intercambio docente con posibilidades de servicio en otros países, incorporar en los sistemas de estudio la dimensión de lo latinoamericano. Solo en esta forma se podrá contrarrestar la dimensión imprevisible de la globalización en el sentido de que la multilateralizacion de las instituciones de poder y la descentralización de la autoridad a los gobiernos regionales y locales producen una nueva geometría de poder. 


\section{Referencias}

Cárdenas, A. et al. (2000). El maestro protagonista del cambio educativo. Bogotá: Magisterio.

Ciencia y Política. (1991 a 2005). Revista trimestral para América latina y España. Colección Completa. Bogotá: s.n.

CNA: Estrategia de Internacionalización del Consejo Nacional de Acreditación (CNA) de Colombia; Bogotá, CNA, junio del 2007.

Colciencias. (s.f.). Ciencia, Tecnología y Desarrollo. Fondo Colombiano de Investigaciones. Colección Completa.

Consejo Nacional de Acreditación [CNA]. (2008). Lineamientos para la acreditación de alta calidad de programas de maestría y doctorado. Bogotá: s.n.

Convenio Andrés Bello. Revista del Convenio Andrés Bello, XII(1-31).

Ministerio de Educación Nacional. (2002). Guía para la Internacionalización de las Instituciones de la Educación Superior. Bogotá: ICFES

Misión Ciencia, Educación y Desarrollo. (1997). Informe conjunto. Colombia: al filo de la oportunidad. Bogotá: IDEP.

Restrepo, R., Sánchez, M. \& Uribe, A. (2006). Aseguramiento de la calidad en los programas de posgrado ( $2^{\mathrm{a}}$ ed.). Antioquia: Universidad de Antioquia.

Universidad Autónoma Latinoamericana. (2005). Revista de ANAULA, (1-25).

Universidad Central. (s.f.). Hojas Universitarias, 3, 4, 5(1-30).

Universidad Pedagógica Nacional. (1993). En nuestra América. Aportes a la historia de la cultura y de la educación. Bogotá: U.P.N.

Universidad Pedagógica Nacional. (2004). Encuentro iberoamericano de formación docente. Tomo I. Bogotá: U.P.N. 